\title{
A Deep Learning Approach for Detecting Liver Cirrhosis from Volatolomic Analysis of Exhaled Breath
}

Mikolaj A Wieczorek

Mayo Clinic

Alexander D Weston

Mayo Clinic

Matthew F Ledenko

Mayo Clinic

Jonathan Nelson Thomas

Mayo Clinic

Rickey E Carter

Mayo Clinic

Tushar C Patel ( $\square$ patel.tushar@mayo.edu )

Mayo Clinic

Research Article

Keywords:

Posted Date: February 2nd, 2022

DOI: https://doi.org/10.21203/rs.3.rs-1258834/v1

License: (c) (1) This work is licensed under a Creative Commons Attribution 4.0 International License.

Read Full License 


\section{Abstract}

\section{Introduction}

Liver disease such as cirrhosis is known to cause changes in the composition of volatile organic compounds (VOC) present in patient breath samples. Previous studies have demonstrated the diagnosis of liver cirrhosis from these breath samples, but studies are limited to a handful of discrete, wellcharacterized compounds.

\section{Methods}

We utilized VOC profiles from breath samples from 46 individuals, 35 with cirrhosis and 11 healthy controls. A deep-neural network was optimized to discriminate between healthy controls and individuals with cirrhosis.

\section{Results}

A 1D convolutional neural network (CNN) was accurate in predicting which patients had cirrhosis with an AUC of 0.90 (95\% Cl: 0.75, 0.99). Shapley Additive Explanations characterized the presence of discrete, observable peaks which were implicated in prediction, and the top peaks (based on the average SHAP profiles on the test dataset) were noted.

\section{Conclusion}

CNNs demonstrate the ability to predict the presence of cirrhosis based on a full volatolomics profile of patient breath samples. SHAP values indicate the presence of discrete, detectable peaks in the VOC signal.

\section{Introduction}

\section{Liver Cirrhosis}

Cirrhosis of the liver is an advanced stage of disease in which the liver is damaged from scarring or fibrosis as a result of chronic hepatic injury that can arise from conditions such as chronic infection with Hepatitis B or C virus, excess alcohol or other causes [1]. Cirrhosis can be classified as compensated or decompensated. A diagnosis of compensated cirrhosis can be challenging as these patients can be asymptomatic and may have normal laboratory or imaging findings.

The presence of cirrhosis can be inferred from clinical, laboratory, radiologic or elastographic findings, but a liver biopsy represents the gold-standard for diagnosis [2]. Cirrhosis is a preneoplastic condition and a major risk factor for hepatocellular carcinoma (HCC) which is the sixth most prevalent cancer and third leading cause of cancer-related death [3]. Once diagnosed, close monitoring for progression as well as active surveillance for onset of HCC are essential [4]. The onset of complications such as ascites, varices 
and hepatic encephalopathy define decompensated cirrhosis, and are associated with a higher risk of death. [5].

\section{Volatile Organic Compounds as disease biomarkers}

Liver disease has long been recognized to be associated with detectable changes in a patient's breath, e.g. fetor hepaticus [6, 7]. These result from the presence of diverse range of Volatile Organic Compounds (VOCs), which may consist of byproducts of liver metabolism that are released into the bloodstream and eventually eliminated in the patient's breath. Importantly, VOCs have been associated with liver cirrhosis [8] and fibrosis [9]. Thus, a reliable detection of disease-associated VOC or other metabolites altered by liver damage have the potential for use as a noninvasive test for the diagnosis and monitoring of cirrhosis.

\section{Global volatolomic analysis-based biomarkers}

Global volatolomic analyses can be performed on exhaled breath samples by separating and detecting individual VOCs. The approaches for detection of individual VOC are laborious and time consuming, and often require the use of sophisticated equipment. A limitation of several prior breath-based biomarker studies is that they rely on identification of a single VOC such as limonene [10], which may miss more complex signatures of disease. The large number and variability of VOC in exhaled breath have hampered the development of individual breath-based biomarkers for disease. Recognizing the inherent variability and diversity of individual VOCs with biomarker potential, we sought to evaluate approaches for an unbiased global volatolomic profiles as disease biomarkers. For our study, global volatolomic profiling was performed using thermal desorption (TD) with gas chromatography (GC) based separation coupled with field asymmetric ion mobility spectrometry (FAIMS) for biomarker discovery.

Analysis of volatolomic profiles has been greatly aided with the use of artificial intelligence (Al) algorithms such as deep convolutional neural networks which can analyze relationships between all detectable compounds represented in a breath sample.

\section{Deep Learning}

This study builds upon existing techniques to diagnose liver cirrhosis from noninvasive breath samples using an artificial neural network based on TD-GC-FAIMS signal. We demonstrate that cirrhosis results in detectable, quantifiable changes in the volatolomic profile of a patient's breath. Furthermore, by utilizing Shapley Additive Explanations, we demonstrate a set of volatolomic features that correspond to disease prediction and reflect biomarkers that can be used for the detection of disease without the need to rely on identification of individual VOCs.

\section{Results}

\section{Patient demographics}


A total of 46 individuals (157 samples) were included in this study (123 samples from 35 patients with decompensated or compensated cirrhosis and 34 control samples from 11 healthy individuals). Among the 46 patients included, median age was 57 (Range: 24 - 76), 35/46 (76\%) had history of liver cirrhosis, $23 / 46$ (50\%) were men. A comparison of demographics between healthy and cirrhosis patients is depicted in Table 1. In comparison to healthy controls, cirrhosis patients had an older age at diagnosis (median: 61 vs. 45 years, $P=0.001)$ and were more likely to be obese ( $51.3 \%$ vs. $27.3 \%$ ).

Within the disease cohort, 14 patients (35.9\%) had stage I cirrhosis, 15 patients (38.5\%) had stage II cirrhosis, and 10 patients (25.6\%) had stage III cirrhosis. A comparison of laboratory test results across cirrhosis stages is shown in Table 2. As expected by the cirrhosis severity classifications, stage III cirrhosis had highest model for end-stage liver disease (MELD), aspartate aminotransferase to platelet ratio index (APRI), and Fibrosis-4 index for liver fibrosis (FIB-4) scores with medians 13, 0.9, 6, respectively. Supplemental Table 1 expands upon Table 2, including additional laboratory test results.

Table 1

Comparison of demographics between healthy and cirrhosis patients.

\begin{tabular}{|llll|}
\hline \multicolumn{4}{|c|}{ Median (minimum, maximum) or No. (\%) of patients } \\
\hline Sex (Male) & Disease $(\mathrm{N}=35)$ & Healthy (N=11) & P value \\
\hline Female & & $6(54.5 \%)$ & 1.00 \\
\hline Male & $17(48.6 \%)$ & $5(45.5 \%)$ & \\
\hline Age (years) & $18(51.4 \%)$ & $45.0(24.0,60.0)$ & $<0.001$ \\
\hline Age group (years) & $61.0(33.0,76.0)$ & & 0.002 \\
\hline $\begin{array}{l}\text { (20,50] } \\
\text { (50,80] }\end{array}$ & $7(20.0 \%)$ & $8(72.7 \%)$ & 0.42 \\
\hline Body mass index (kg/m2) & $28(80.0 \%)$ & $3(27.3 \%)$ & 0.20 \\
\hline Body mass index (categorical) & $30.2(20.2,41.3)$ & $27.6(21.0,41.8)$ & \\
\hline Healthy weight (18.5-24.9) & $5(14.3 \%)$ & & \\
\hline Overweight (25.0-29.9) & $10(28.6 \%)$ & $6(54.5 \%)$ & \\
\hline Obesity (>30.0) & $20(57.1 \%)$ & $3(27.3 \%)$ & \\
\hline $\begin{array}{l}\text { P-values result from a Wilcoxon rank sum test (continuous variables) or Fisher's exact test } \\
\text { (categorical variables). }\end{array}$ & & & \\
\hline
\end{tabular}


Table 2

Comparison of characteristics across disease stage for the cirrhosis study population.

\section{Median (minimum, maximum) or No. (\%) of patients}

\begin{tabular}{|c|c|c|c|c|}
\hline & $\begin{array}{l}\text { Cirrhosis Stage } I_{1} \\
\text { Compensated }(\mathrm{N}=13)\end{array}$ & $\begin{array}{l}\text { Cirrhosis Stage II, } \\
\text { Compensated }(\mathrm{N}=12)\end{array}$ & $\begin{array}{l}\text { Cirrhosis Stage III, } \\
\text { Decompensated }(\mathrm{N}=10)\end{array}$ & $\begin{array}{l}\mathrm{P} \\
\text { value }\end{array}$ \\
\hline Ascites & $0(0.0 \%)$ & $0(0.0 \%)$ & $10(100.0 \%)$ & \\
\hline Varices & $0(0.0 \%)$ & $12(100.0 \%)$ & $8(80.0 \%)$ & \\
\hline Platelets & $185.0(123.0,272.0)$ & $92.5(44.0,279.0)$ & $83.0(36.0,238.0)$ & 0.014 \\
\hline MELD & $8.0(6.0,20.0)$ & $10.0(7.0,19.0)$ & $13.0(7.0,28.0)$ & 0.041 \\
\hline APRI & $0.4(0.2,1.1)$ & $0.8(0.2,3.5)$ & $0.9(0.3,3.5)$ & 0.16 \\
\hline FIB4 & $2.4(0.6,4.2)$ & $3.7(1.2,10.7)$ & $6.0(1.3,14.8)$ & 0.013 \\
\hline
\end{tabular}

\section{Model performance at the sample and patient levels}

The CNN model was successful in differentiating breath samples taken from patients with cirrhosis versus healthy controls; four models trained on separate CV splits classified the presence of cirrhosis with an average AUC of 0.79 at the sample level (clustering between technical replicates precludes accurate estimation of the exact $95 \% \mathrm{Cl}$ at the sample level; these values are reported for the primary endpoint of patient diagnosis only). When these models were combined into an ensemble by averaging prediction probabilities, the AUC was 0.90 as depicted by Figure 1 .

At the patient level, the ensemble model prediction outperformed the four constituent CV models in detecting the presence of cirrhosis in patients. Individual models discriminated between cirrhosis individuals and healthy controls with an average AUC of 0.80 (range: $0.54,1.00$ ), their ensemble achieved an AUC of 0.90 (95\% Cl: $0.75,1.00)$. At a $50 \%$ classification threshold, the ensemble model yielded the following performance metrics: sensitivity of 1.00 (perfect), specificity of 0.40 , and F1 score of 91.9 .

All diagnostic performance measures for the ensemble and its constituent CV models are reported in Table 3 at both sample and patient levels. The subgroup analysis did not reveal any significant differences in model performance between subgroups (age, BMI, or sex). 
Table 3

Model performance metrics at sample and patient levels at the 0.5 threshold. $95 \%$ Confidence Intervals are reported at the patient level only, clustering of technical replicates precluded calculation of the exact confidence interval at the sample level.

\begin{tabular}{|c|c|c|c|c|c|}
\hline & $\begin{array}{l}\text { AUC } \\
(95 \% \mathrm{Cl})\end{array}$ & $\begin{array}{l}\text { Accuracy } \\
(95 \% \mathrm{Cl}) \text {, fraction }\end{array}$ & $\begin{array}{l}\text { Sensitivity } \\
(95 \% \mathrm{Cl}) \text {, fraction }\end{array}$ & $\begin{array}{l}\text { Specificity } \\
(95 \% \mathrm{Cl}), \\
\text { fraction }\end{array}$ & $\begin{array}{l}\text { F1 } \\
\text { Score }\end{array}$ \\
\hline \multicolumn{6}{|l|}{$\begin{array}{l}\text { Sample } \\
\text { level }\end{array}$} \\
\hline Ensemble & 0.899 & $86.7 \% 65 / 75$ & $100.0 \% 59 / 59$ & $37.5 \% 6 / 16$ & 92.2 \\
\hline CV1 & 0.800 & $86.7 \% 65 / 75$ & $100.0 \%$ 59/59 & $37.5 \% 6 / 16$ & 92.2 \\
\hline CV2 & 0.890 & $85.3 \% 64 / 75$ & $93.2 \% 55 / 59$ & $56.2 \% 9 / 16$ & 90.9 \\
\hline CV3 & 0.771 & $85.3 \% 64 / 75$ & $98.3 \% 58 / 59$ & $37.5 \% 6 / 16$ & 91.3 \\
\hline CV4 & 0.682 & $81.3 \% 61 / 75$ & $93.2 \% 55 / 59$ & $37.5 \% 6 / 16$ & 88.7 \\
\hline \multicolumn{6}{|l|}{$\begin{array}{l}\text { Patient } \\
\text { level }\end{array}$} \\
\hline Ensemble & $\begin{array}{l}0.894 \\
(0.751 \\
1.000)\end{array}$ & $\begin{array}{l}86.4 \%(65.1 \%, \\
97.1 \%) 19 / 22\end{array}$ & $\begin{array}{l}100.0 \%(80.5 \% \\
100.0 \%) 17 / 17\end{array}$ & $\begin{array}{l}40.0 \%(5.3 \% \\
85.3 \%) 2 / 5\end{array}$ & 91.9 \\
\hline CV1 & $\begin{array}{l}0.824 \\
(0.627 \\
1.000)\end{array}$ & $\begin{array}{l}86.4 \%(65.1 \% \\
97.1 \%) 19 / 22\end{array}$ & $\begin{array}{l}100.0 \%(80.5 \% \\
100.0 \%) 17 / 17\end{array}$ & $\begin{array}{l}40.0 \%(5.3 \% \\
85.3 \%) 2 / 5\end{array}$ & 91.9 \\
\hline cV2 & $\begin{array}{l}0.882 \\
(0.691 \\
1.000)\end{array}$ & $\begin{array}{l}81.8 \%(59.7 \% \\
94.8 \%) 18 / 22\end{array}$ & $\begin{array}{l}88.2 \%(63.6 \% \\
98.5 \%) 15 / 17\end{array}$ & $\begin{array}{l}60.0 \%(14.7 \% \\
94.7 \%) 3 / 5\end{array}$ & 88.2 \\
\hline cV3 & $\begin{array}{l}0.800 \\
(0.486 \\
1.000)\end{array}$ & $\begin{array}{l}86.4 \%(65.1 \%, \\
97.1 \%) 19 / 22\end{array}$ & $\begin{array}{l}100.0 \%(80.5 \% \\
100.0 \%) 17 / 17\end{array}$ & $\begin{array}{l}40.0 \%(5.3 \% \\
85.3 \%) 2 / 5\end{array}$ & 91.9 \\
\hline CV4 & $\begin{array}{l}0.682 \\
(0.371 \\
0.994)\end{array}$ & $\begin{array}{l}81.8 \%(59.7 \%, \\
94.8 \%) 18 / 22\end{array}$ & $\begin{array}{l}94.1 \%(71.3 \% \\
99.9 \%) 16 / 17\end{array}$ & $\begin{array}{l}40.0 \%(5.3 \% \\
85.3 \%) 2 / 5\end{array}$ & 88.9 \\
\hline
\end{tabular}

\section{Performance based on the cirrhosis stage}

At the $50 \%$ threshold, the model correctly classified $100 \%$ of patients with stage I, stage II, or stage III cirrhosis (17/17 patients; 59/59 samples), i.e., the model achieved perfect sensitivity. The model correctly identified $2 / 5$ healthy individuals (6/16 healthy samples) but incorrectly classified $3 / 6$ healthy individuals (10/16 healthy samples) as having cirrhosis (Figure 1). Evaluation of the ensemble model across the severity of cirrhosis at both the sample and patient levels is shown (Figure 2). The model displayed the highest confidence in predicting stage II and stage III cirrhosis (median probabilities $>0.99$ ) but 
performed relatively lower in differentiating patients with stage I cirrhosis from healthy controls (median probability >0.76).

\section{Identification of TD-GC-FAIMS features}

The SHAP values which identify peaks in the signal that contributed most to the prediction are depicted by the beeswarm summary plots in Figure 3. For each CV model, we identified the top ten peaks which selected a total of 22 unique compounds in the TD-GC-FAIMS signal; 14 compounds (64\%) were identified by at least two independently trained CV models, two compounds were identified by three CV models, and one compound was identified by all four CV models. Figure 4 displays an example of four patients (one from each severity stage of cirrhosis and one healthy control) whose VOC profiles' signal is visualized with overlaying heatmaps, which depict the five most important compounds in the classification of liver cirrhosis identified by each model.

\section{Discussion}

This work presents a deep-learning based approach for detecting liver cirrhosis based on noninvasive breath samples analyzed with TD-GC-FAIMS. To our knowledge, this is the first application of deep-neural networks for the prediction of liver cirrhosis from volatolomic profiles from patient breath samples [23 26]. We observed that convolutional neural networks were an effective technique for analyzing the volatolomic profiles obtained using TD-GC-FAIMS, and our optimal model displayed an AUC of 0.90 and an accuracy of $86 \%$ at the patient level. This supports the application of volatolomic analyses using TDGC-FAIMS for noninvasive diagnosis of cirrhosis from breath samples.

\section{Deep learning approach}

Several deep-learning approaches were attempted including transfer-learning of a pretrained ResNet, and a fully-connected deep neural network. Previous experiments done by this group have demonstrated machine-learning based approaches for the detection of cirrhosis [19]. We observed optimal performance with a convolutional neural network model. This is consistent with extensive literature that indicates CNNs are an efficient and accurate method of analyzing sparse signals data; in the medical field, CNNs are popular model for both image analysis and signals processing [27 - 31].

Optimal performance was observed with an ensemble of four CNNs combined by taking the mean prediction probability; the ensemble performance was slightly better than the best performing constituent models, and substantially better than the average of its four constituents. Combining several models into an ensemble is an effective technique for generating consistent predictions and reducing the impact of overfitting.

\section{Model performance in severe cirrhosis}

Our model was effective in predicting the presence of cirrhosis with an accuracy of $86 \%$ at the patient level. The model displayed a tendency to overdiagnose the presence of cirrhosis; the ensemble model had a sensitivity of $100 \%$ but a specificity of $40 \%$ at the patient level. 
At the sample level, all mistakes came from differentiating healthy controls from patients with stage I cirrhosis (e.g. the lowest stage of disease, when individuals are often asymptomatic). This suggests that the model is correctly identifying hallmarks of severe disease with a very high level of accuracy.

Imbalance in the training dataset likely played a role in model specificity (only 11/46 individuals included in this study were healthy controls). Specificity may be modified by adjusting the prediction cutoff from 0.5 to a higher value, with the understanding that this may increase the rate of false negatives. $A$ diagnostic tool with high sensitivity could be appropriate as an inexpensive, non-invasive screening tool for cirrhosis detection in an at-risk population, with the understanding that additional diagnostic tests such as imaging exams would be required to rule out false positive results in an initial screen.

\section{Explainable Al}

The use of SHAP for explaining the predictions of the CNN model identified several discrete peaks which were consistently associated with either a positive or negative prediction. We observed that $14 / 22$ (63\%) of the top peaks detected by the ensemble model were identified by multiple independent CV models, which indicates that these features are reproducible between independently trained models. This supports the accuracy of the CNN approach.

Several specific VOCs are known to be overexpressed or underexpressed in cirrhotic patients, including limonene, methanol, and 2-pentanone [8]. Data-driven approaches such as deep neural networks rely on the entire volatolomic profile measurements, not only a few discrete peaks, and therefore may be incorporating VOCs which have not yet been identified, or VOC constituents which are partially metabolized from known compounds. Future work has the potential to characterize previously unknown VOCs which the model indicates are implicated in cirrhosis.

\section{Limitations}

We acknowledge several important limitations to this study. This is a single site, preliminary study in a modest dataset of 46 patients (157 samples), 35 (76\%) of which had cirrhosis. The study cohort encompasses a broad range of diseases of several etiologies that may manifest in different volatolomic signatures of metabolic effects.

In several instances, model performance is reported across technical replicates to demonstrate the reproducibility of the TD-GC-FAIMS technique. Clustering between technical replicates precludes accurate assessment of the $95 \%$ confidence interval for several metrics including the ROC curve. The primary outcome remains prediction of cirrhosis at the patient level by our ensemble model, and $95 \%$ confidence intervals are provided for patient predictions when appropriate.

This relatively high disease burden likely impacted the specificity of the model despite techniques such as data augmentation, downsampling, and model ensembling aimed at preventing overfitting. Ongoing subject recruitment focuses on the collection of additional healthy controls. Strong performance on the test dataset between constituent models, and in cases of severe cirrhosis, coupled with reproducibility of 
features from SHAP analysis, support the conclusion that the model is identifying clear, tangible VOC signals even on a modest dataset.

\section{Future Direction}

This preliminary study reports a CNN model with very high sensitivity to detecting cirrhosis (sensitivity of $100 \%$ at the patient level). Ongoing study enrollment will focus on sample collection from healthy individuals with the goal to increase the specificity of the model.

Additionally, higher specificity models could be developed to help counteract the susceptibility to type I error (i.e. false-positive classifications), for example, by training a multiclass model to differentiate between stage I, stage II, and stage III cirrhosis, or by directly predicting a metric such as the FIB-4 score. This could potentially expand the concept of our early screening Al tool to disease-severity monitoring tool.

Finally, analysis with SHAP indicate the presence of several peaks in the signal which are useful for model prediction, e.g. peaks located at indices 1038 (identified by all four CV models) and indices 1963 and 1216 (identified by three CV models). Discrete peaks validated on a larger sample cohort could provide a target for future characterization of VOCs, targeting compounds which are known to be relevant for human disease.

\section{Conclusion}

A deep learning model is capable of detecting the presence of cirrhosis in volatolomic profiles obtained from analyses of exhaled breath samples from patients using TD-GC-FAIMS. Model performance had an AUC of 0.90 and a sensitivity in detecting disease of $100 \%$ at the patient level. Use of SHAP as a technique for explainable Al detected a set of unique peaks associated with both positive and negative prediction; $63 \%$ of the top ten peaks were reproducible across multiple independently trained models. This technique demonstrates feasibility of a noninvasive clinical screening exam for diagnosing and monitoring liver cirrhosis from noninvasive breath samples without the need for detection and characterization of individual metabolites.

\section{Materials And Methods}

\section{Study participants}

This prospective study was conducted under a Mayo Clinic institutional review board (IRB) approved protocol. All methods utilized in this study for data collection were carried out in accordance with relevant guidelines and regulations. Informed consent was obtained from study participants in writing. The trial is registered at clinicaltrials.gov (NCT04341012), first registration on 10/04/2020.

All participants in this prospective study were enrolled between September 2019 and March 2020. The study inclusion criteria were the ability to provide informed consent and age greater than 18 years. 
Participants were categorized into groups based on absence or presence of cirrhosis and/or portal hypertension, and of individual complications as determined on clinical bases which included histologic, clinical, laboratory, or imaging features. Participants with non-cirrhotic portal hypertension were excluded.

\section{Variable definitions}

A clinical diagnosis of cirrhosis served as our ground truth training label and reference standard. Cirrhosis was classified as stage I, stage II, or stage III. Stage I was defined as compensated cirrhosis with the absence of varices or other clinical complications. Stage II (compensated) cirrhosis was defined as presence of varices but no other complications. The presence of varices in patients with compensated cirrhosis is a prognostic factor and indicates a higher risk of decompensation. Stage III (decompensated) cirrhosis was defined as the presence of ascites, variceal hemorrhage, or hepatic encephalopathy. Diagnoses of cirrhosis and presence of clinical complication were determined independently by two hepatologists

\section{Sample collection}

A flow-chart of sample collection and volatolomic analysis is shown in Figure 5. Each study participant provided a single breath sample collected using the ReCIVA breath sampler (Owlstone Medical, Cambridge, UK) and passed through thermal desorption tubes to capture VOCs, then separated using high temperatures and gas chromatography (GC) and passed onto FAIMS (Owlstone Medical, Cambridge, UK), a spectrometry device which separates ions based on size and charge to create a data matrix that represents a volatolomic profile of the breath sample [11]. FAIMS has been used for VOC detection in many settings [12 - 18].

Data collection using this approach (TD-GC-FAIMS) has been described previously [19]. Each study participant provided a breath sample totaling to 1-liter of exhaled air onto Bio-Monitoring TD tubes (Markes International, South Wales, United Kingdom). Samples were divided into four technical replicates which were derived from the same $1 \mathrm{~L}$ breath sample and were collected simultaneously on four separate collection tubes by the collection mask. Samples were collected by a trained technician (J.T.) after patients had fasted at least four hours from food or drink besides water. A subset of 11 samples from 11 individuals (1 sample per individual) had been stored for a period of time exceeding 6 weeks; these samples were excluded from the analysis because the effects of long-term cold storage on breath VOCs are poorly understood $[20,21]$.

\section{Analysis of Volatile Organic Compounds}

The TD-GC-FAIMS data output was preprocessed to separate the ion intensities from each dispersion field (DF) setting and subtract out environmental VOCs and background current fluctuations using air filter field control blanks. All technical replicates were analyzed independently. The negative and positive ion intensity mesh matrices at each respective DF were combined and outer matrix cells with intensity values below the overall maximum baseline intensity $(0.0104 \mathrm{pA})$ were removed. Compensation field (CF) scan points were limited to between $-3 \mathrm{~V}$ and $+3 \mathrm{~V}$. In addition, 30 terminal time resolved values, approximately 
40 seconds at the end of the gas chromatography run, were removed for each DF data matrix. Data preprocessing was conducted in Matlab version 2019b (MathWorks, Matick, MA).

For purposes of training deep-learning models, outputs were additionally processed by dividing by the maximum value. The signal was downsampled from a $2 \mathrm{D}$ to a $1 \mathrm{D}$ signal by taking the maximum value for each row. The final output of the workflow was a signal with 3,400 rows (e.g. could capture up to 3,400 unique molecular features). During model training, data were randomly augmented with $5 \%$ Gaussian random noise.

\section{Training of the deep learning model}

For model evaluation purposes, all samples from 22 individuals (totaling 75 samples), including 17 positive patients ( 59 samples) and 5 healthy controls ( 16 samples) were randomly selected and set aside as a test dataset; these samples were excluded from the model development process. The dataset was split at the patient level such that no patients had samples in both the training and test dataset. The 24 individuals (82 samples), which included 18 positive patients ( 64 samples) and 6 healthy controls (18 samples) were used for training and validating the neural network model. The ground truth label was taken to be the presence of cirrhosis as determined by clinical experts. All results are reported on the test dataset.

The 24 individuals were randomly divided into four splits using stratified group 4-fold cross-validation; each split consisted of three analysis folds and one assessment fold where the analysis folds were used for training and the assessment fold was used for validating the model. The same cross-validation (CV) split was used for all iterations of hyperparameter tuning. Partitioning was done at the patient level such that no individual had samples in more than a single CV fold. Although partitioning patients into each fold was random, we attempted to preserve the distribution of our outcome with stratification, where at least one healthy patient was represented in each CV split; this was necessary to ensure proper training of the model. Supplemental Table 2 and Supplemental Figure 1 provide details on the data partition.

\section{Model development}

Several potential deep learning model architectures were evaluated to predict presence of cirrhosis. We applied a pretrained ResNet, a custom fully-connected deep-neural network, and a custom convolutional neural network (CNN). The CNN outperformed other models during this initial experiment and was selected for the architecture and hyperparameter tuning process.

The CNN model architecture and hyperparameters were optimized through grid search. Parameters which were considered included the number of convolutional layers, number of kernels per layer, number of fully-connected ("dense") layers at the output end of the model, learning rate, batch size as well as alternate methods of augmenting the data to account for data imbalance. Figure 6 displays the architecture of the best-performing CNN model discovered through hyperparameter tuning. Supplemental Table 3 lists all parameters considered. Additionally, model architecture and hyperparameter optimization 
grid search results were summarized with analysis (training) and assessment (validation) accuracy and loss curves in an interactive $R$ markdown document ( $R$ version 4.0.3 with Shiny 1.6.0).

The optimal model architecture and hyperparameter configuration was selected by assessing the average highest performing validation accuracy and lowest validation loss across all four CV splits. The bestperforming four individual CNN models, one for each corresponding CV split, were combined into an ensemble model by taking the average of model outputs.

Model development with hyperparameter tuning was performed on the Google Cloud Platform (GCP) and was accelerated using 1 Nvidia T4 GPU (16GB RAM).

\section{Model evaluation}

The primary endpoint of this study was diagnosis of liver cirrhosis at the patient level, which was achieved by combining the four individual constituent CNN models from four cross-validation splits (CV1, CV2, CV3, CV4) into an "ensemble" prediction by taking the mean probability across all four models, and then by taking the median value of this ensemble prediction across the $3-4$ technical replicates per patient. Additionally, to evaluate the reproducibility of model prediction across multiple technical replicates, the AUC curve and summary metrics are reported at the sample level.

To investigate the algorithm's ability to discriminate cirrhosis patients from healthy controls within different severity levels, the ensemble model's predicted probabilities were tabulated by cirrhosis stage and visualized with boxplots.

\section{Model explainability}

Interpretation of artificial intelligence algorithms is an increasingly important approach to validate their performance and lend insight to the modeling process. To aid in the interpretation of the results of the CNN, we utilized Shapley Additive Explanations (SHAP) to determine which features contribute to the detection of liver disease [22]. SHAP identifies features which are important in determining the model output by allocating contributions of the model output across input parameters. SHAP was implemented in the SHAP package version 0.39.0 for Python 3.7.8. SHAP values were computed individually for the four CNN models.

SHAP feature importance plots were summarized on the training and test datasets for each CV split with "beeswarm" scatter plots [22]. To identify individual compounds from TD-GC-FAIMS which are most important for detecting the presence of liver disease, the five features with the largest magnitude (largest absolute SHAP value) were selected per each instance in the test dataset ( 75 samples) and were overlayed on the sample's VOC signal, creating "heatmaps" which identify peaks important for predictions. The heatmaps were visualized with darker red representing the higher number of times the same peak was detected across four constituent models. For each patient, the final ensemble predicted probability was annotated.

\section{Statistical analysis}


Clinical demographics and laboratory test results data were summarized with the median and range for the continuous variables and with the number and percentage of patients for the categorical variables. The Wilcoxon rank sum test for continuous variables and Fisher's exact test for categorical variables were used to compare demographics between healthy controls and cirrhosis patients; Kruskal-Wallis rank sum test was used to compare laboratory test results between stage I, stage II, and stage III cirrhosis.

Model performance was assessed for the four individual cross-validated models as well as the ensemble model at the sample and patient levels using the area under the receiver operating characteristic curve (AUC), accuracy, sensitivity, specificity, and F1 score. A threshold cutoff value of $>0.50$ was used to classify a sample or patient as positive (presence of any stage of cirrhosis). The exact $95 \%$ confidence intervals were computed for AUC, accuracy, sensitivity, and specificity metrics at the patient level (Pearson-Klopper method).

To explore patterns in patient subgroups, subgroup analysis was performed on the final ensemble model with respect to age, $\mathrm{BMI}$, and sex at the patient level.

Model development and hyperparameter tuning were performed using Tensorflow version 2.3.0 for Python version 3.7.8. Data summaries, statistical analysis, visualizations, and model evaluation were performed using R Statistical Software (version 4.0.3; R Foundation for Statistical Computing, Vienna, Austria

\section{Declarations}

\section{Data Availability Statement}

Data cannot be shared publicly because of patient privacy concerns. Data are available for researchers who meet the criteria for access to confidential data following approval by the Mayo Clinic IRB (www.mayo.edu). De-identified data can be requested through a Materials Transfer Agreement. Requests for patient-related data that is not included in the paper will not be considered. All requests for data will be reviewed by the Mayo Clinic Ventures, Business Development and Legal departments to verify whether the request is subject to any confidentiality restrictions or constitutes intellectual property.

The interactive R-markdown document detailing model performance will be made publicly available. This includes the model architecture, hyperparameters, and loss metrics. The use of an R-markdown document is chosen to aid in understandability while maintaining institutional guidelines for minimizing the risk of $\mathrm{PHI}$ exposure.

\section{Author Contributions Statement}

Authors M.W., A.W., and R.C. prepared the main manuscript text and all figures and tables. Authors M.L., J.T. and T.P. were responsible for technical aspects of analysis as well as being involved in the writing process. All authors reviewed the manuscript. 


\section{References}

1. Tsochatzis, E. A., Bosch, J., \& Burroughs, A. K. (2014). Liver cirrhosis. The Lancet, 383(9930), 17491761.,

2. Kim, M. Y., Jeong, W. K., \& Baik, S. K. (2014). Invasive and non-invasive diagnosis of cirrhosis and portal hypertension. World Journal of Gastroenterology: WJG, 20(15), 4300.

3. Bruix, J., Gores, G. J., \& Mazzaferro, V. (2014). Hepatocellular carcinoma: clinical frontiers and perspectives. Gut, 63(5), 844-855.

4. Mansour, D., \& McPherson, S. (2018). Management of decompensated cirrhosis. Clinical Medicine, 18(Suppl 2), s60.

5. Bruix, Jordi, Maria Reig, and Morris Sherman. "Evidence-based diagnosis, staging, and treatment of patients with hepatocellular carcinoma." Gastroenterology 150.4 (2016): 835-853.

6. Tangerman, A., Meuwese-Arends, M., \& Jansen, J. M. (1994). Cause and composition of foetor hepaticus. The Lancet, 343(8895), 483.

7. Kitiyakara, Taya, et al. "The detection of hepatocellular carcinoma (HCC) from patients' breath using canine scent detection: a proof-of-concept study." Journal of breath research 11.4 (2017): 046002.

8. Del Río, R. Fernández, et al. "Volatile biomarkers in breath associated with liver cirrhosiscomparisons of pre-and post-liver transplant breath samples." EBioMedicine 2.9 (2015): 1243-1250.

9. Fierbinteanu-Braticevici, Carmen, et al. "Role of $13 \mathrm{C}$ methacetin breath test for non invasive staging of liver fibrosis in patients with chronic hepatitis C." The Indian journal of medical research 140.1 (2014): 123.

10. O’Hara, M. E., Del Río, R. F., Holt, A., Pemberton, P., Shah, T., Whitehouse, T., \& Mayhew, C. A. (2016). Limonene in exhaled breath is elevated in hepatic encephalopathy. Journal of breath research, 10(4), 046010.

11. Kolakowski, B. M., \& Mester, Z. (2007). Review of applications of high-field asymmetric waveform ion mobility spectrometry (FAIMS) and differential mobility spectrometry (DMS). Analyst, 132(9), 842864.

12. Ishii, Yukimoto, et al. "Evaluation of pancreatic exocrine secretion using 13C-dipeptide (benzoyl-Ltyrosyl-[1-13C] alanine) breath test: focusing on pancreatoduodenectomy cases." Pancreas 35.4 (2007): 313-319.

13. Beccaria, M., Bobak, C., Maitshotlo, B., Mellors, T. R., Purcaro, G., Franchina, F.A., ... Hill, J. E. (2018). Exhaled human breath analysis in active pulmonary tuberculosis diagnostics by comprehensive gas chromatography-mass spectrometry and chemometric techniques. Journal of breath research, 13(1), 016005.

14. Mellors, T. R., Nasir, M., Franchina, F. A., Smolinska, A., Blanchet, L., Flynn, J.L., ... Hill, J. E. (2018). Identification of Mycobacterium tuberculosis using volatile biomarkers in culture and exhaled breath. Journal of breath research, 13(1), 016004. 
15. Abderrahman, B. (2019). Exhaled breath biopsy: a new cancer detection paradigm. Future Oncology, 15(15), 1679-1682.

16. Lawson, Jonathan, Billy Boyle, and Jonathan Beauchamp. "Driving progress in exhaled breath biomarkers: Breath Biopsy Conference 2019." Journal of Breath Research 14.3 (2020): 030202.

17. Hanna, G. B., Boshier, P. R., Markar, S. R., \& Romano, A. (2019). Accuracy and methodologic challenges of volatile organic compound-based exhaled breath tests for cancer diagnosis: a systematic review and meta-analysis. JAMA oncology, 5(1), e182815-e182815.

18. Trivedi, D. K., Sinclair, E., Xu, Y., Sarkar, D., Walton-Doyle, C., Liscio, C., ...Barran, P. (2019). Discovery of volatile biomarkers of Parkinson's disease from sebum.ACS central science, 5(4), 599-606.

19. Thomas, Jonathan N., Joanna Roopkumar, and Tushar Patel. "Machine learning analysis of volatolomic profiles in breath can identify non-invasive biomarkers of liver disease: A pilot study." PloS one 16.11 (2021): e0260098.

20. Woolfenden, E. (2010). Sorbent-based sampling methods for volatile and semi-volatile organic compounds in air: Part 1: Sorbent-based air monitoring options. Journal of Chromatography A, $1217(16), 2674-2684$.

21. Woolfenden, E. (2010). Sorbent-based sampling methods for volatile and semi-volatile organic compounds in air. Part 2. Sorbent selection and other aspects of optimizing air monitoring methods. Journal of Chromatography A, 1217(16), 2685-2694.

22. Lundberg, S. M., \& Lee, S. I. (2017, December). A unified approach to interpreting model predictions. In Proceedings of the 31st international conference on neural information processing systems (pp. 4768-4777).

23. Pijls, K. E., Smolinska, A., Jonkers, D. M., Dallinga, J. W., Masclee, A. A., Koek, G. H., \& van Schooten, F. J. (2016). A profile of volatile organic compounds in exhaled air as a potential non-invasive biomarker for liver cirrhosis. Scientific reports, 6(1), 1-8.

24. Sinha, R., Lockman, K. A., Homer, N. Z., Bower, E., Brinkman, P., Knobel, H. H., ...Plevris, J. N. (2020). Volatomic analysis identifies compounds that can stratify non-alcoholic fatty liver disease. JHEP Reports, 2(5), 100137.

25. Ferrandino, G., Orf, I., Smith, R., Calcagno, M., Thind, A. K., Debiram-Beecham, I.,... Snowdon, V. K. (2020). Breath Biopsy Assessment of Liver Disease Using an Exogenous Volatile Organic Compound -Toward Improved Detection of Liver Impairment. Clinical and translational gastroenterology, 11(9).

26. Patnaik, R. K., Lin, Y. C., Agarwal, A., Ho, M. C., \& Yeh, J. A. (2021). A Pilot Study for the Prediction of Liver Function Related Scores Using Breath Biomarkers and Machine Learning.

27. Erickson, B. J. (2019). Magician's corner: 2. Optimizing a simple image classifier.

28. Huber, N. R., Missert, A. D., \& Erickson, B. J. (2020). Magician's Corner: 7. Using Convolutional Neural Networks to Reduce Noise in Medical Images.

29. Erickson, B. J., \& Cai, J. (2020). Magician's corner: 4. image segmentation with U-Net. 
30. Nejedly, P., Kremen, V., Sladky, V., Nasseri, M., Guragain, H., Klimes, P., ... Worrell,G. A. (2019). Deeplearning for seizure forecasting in canines with epilepsy. Journal of neural engineering, 16(3), 036031.

31. Attia, Z. I., Noseworthy, P. A., Lopez-Jimenez, F., Asirvatham, S. J., Deshmukh, A.J., Gersh, B. J., ... Friedman, P. A. (2019). An artificial intelligence-enabled ECG algorithm for the identification of patients with atrial fibrillation during sinus rhythm: a retrospective analysis of outcome prediction. The Lancet, 394(10201), 861-867.

32. Kingma, D. P., \& Ba, J. (2014). Adam: A method for stochastic optimization. arXiv preprint arXiv:1412.6

\section{Figures}

A

- $\mathrm{cv}^{1}-\mathrm{cv} 2-\mathrm{cv} 3-\mathrm{cv} 4-$ ensemble

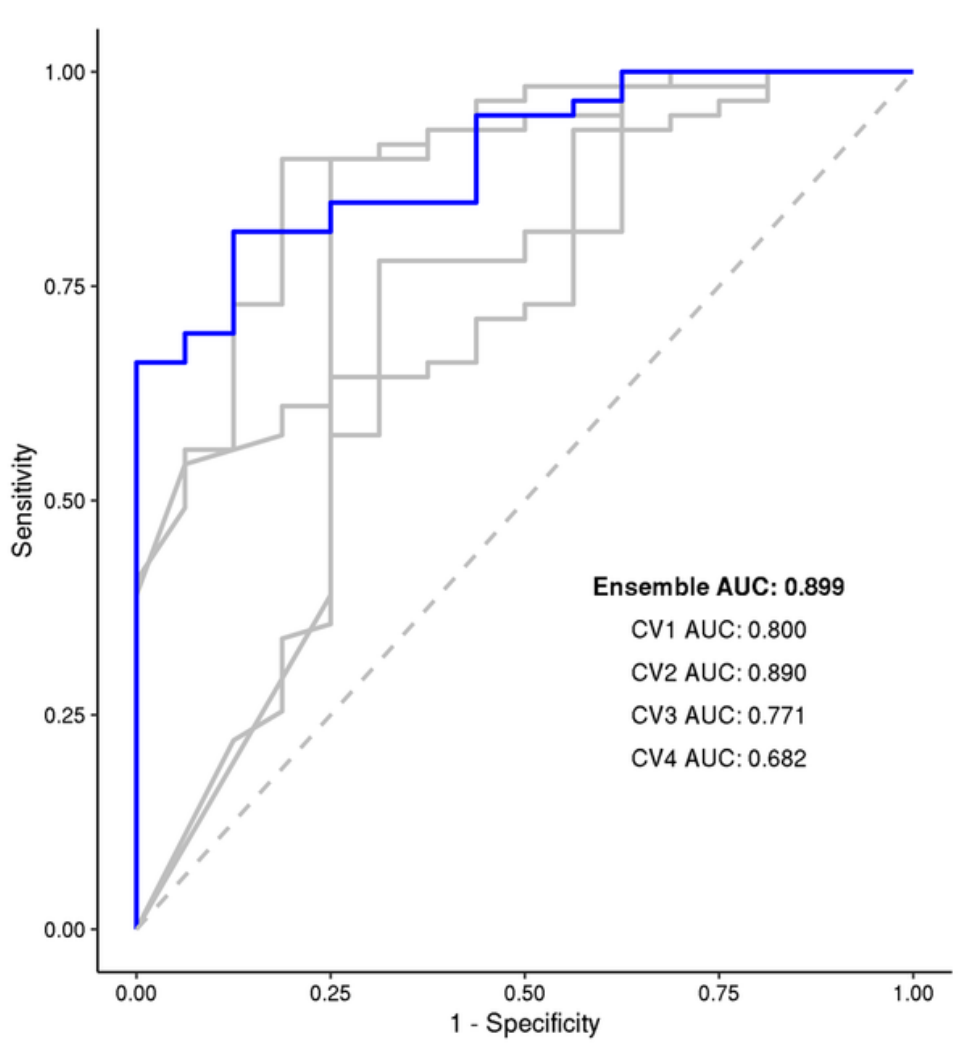

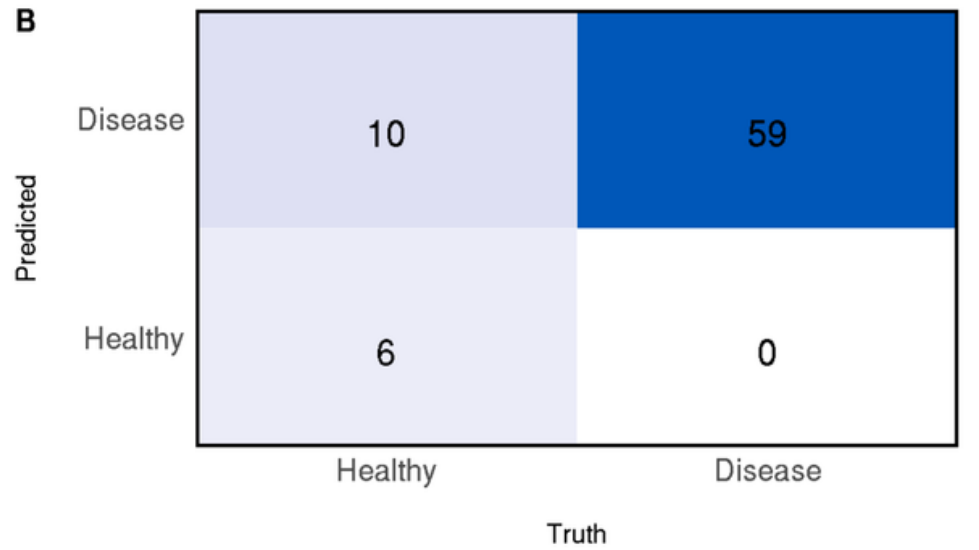

C

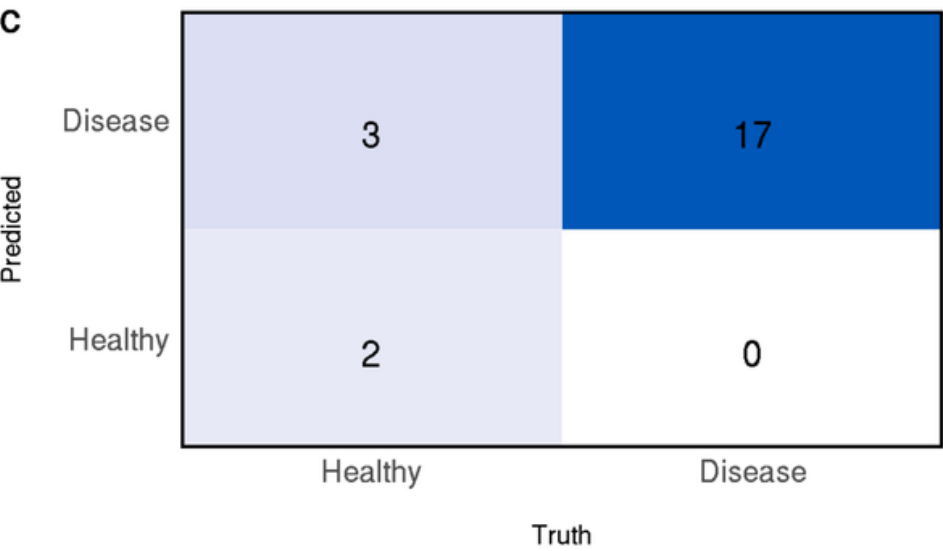

\section{Figure 1}

(A) Receiver operating characteristic (ROC) curves of the final models at the sample level. Area under the ROC curve (AUC) is annotated for each model. The ensemble's confusion matrix heatmaps at the sample (B) and patient (C) levels summarize the frequency of True Positives (TP), False Negatives (FN), True Negatives (TN), False Positives (FP). 

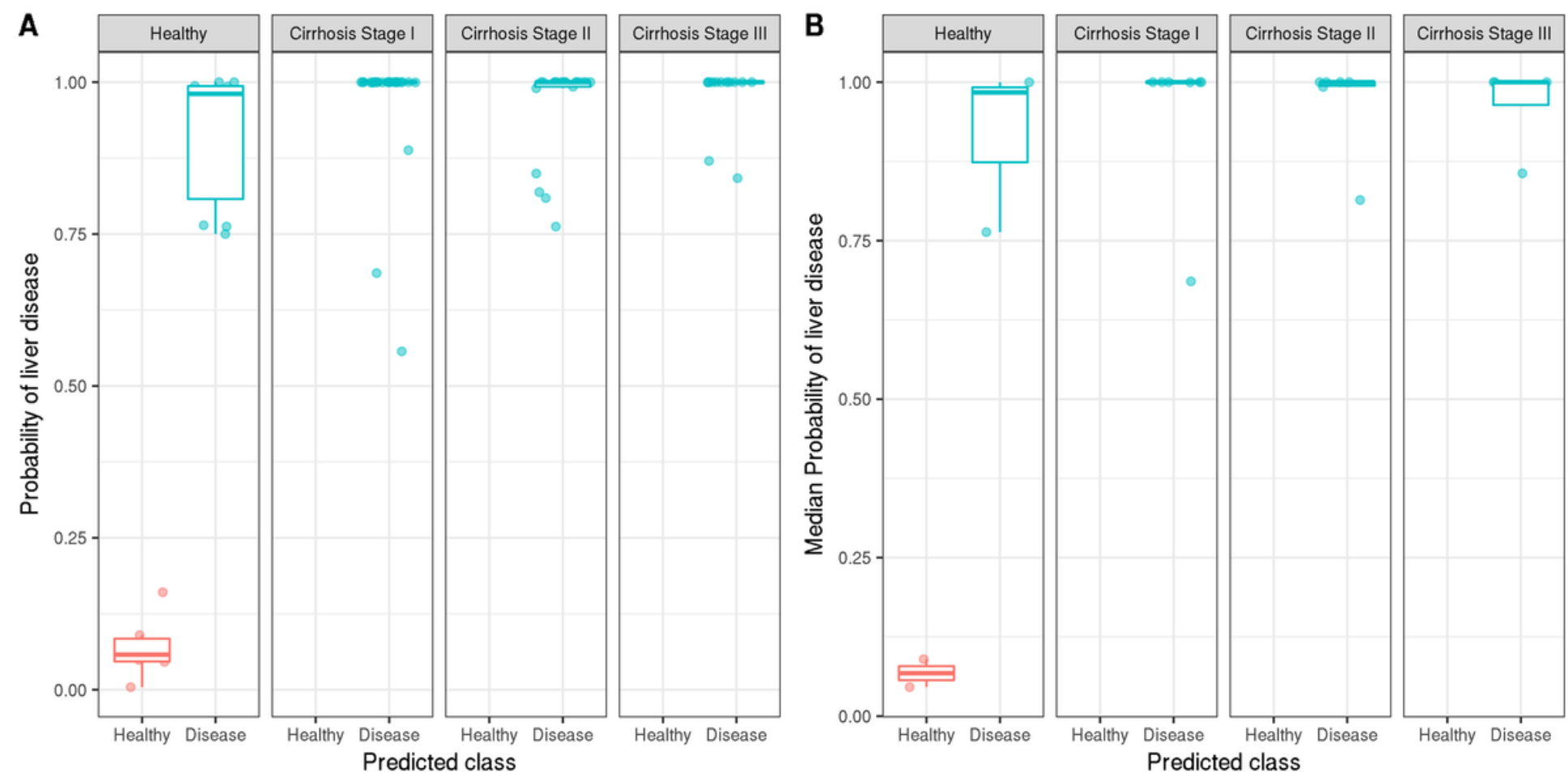

Figure 2

Distribution of the ensemble model's predicted probabilities for healthy vs. disease classifications stratified by the true stage of cirrhosis. Ground truth labels of healthy (red) and disease (blue) are displayed. On the y-axis, probability values of model output are displayed. Model performance is reported at the sample level (A), as well as patient level (B) by aggregating based on median probabilities. 

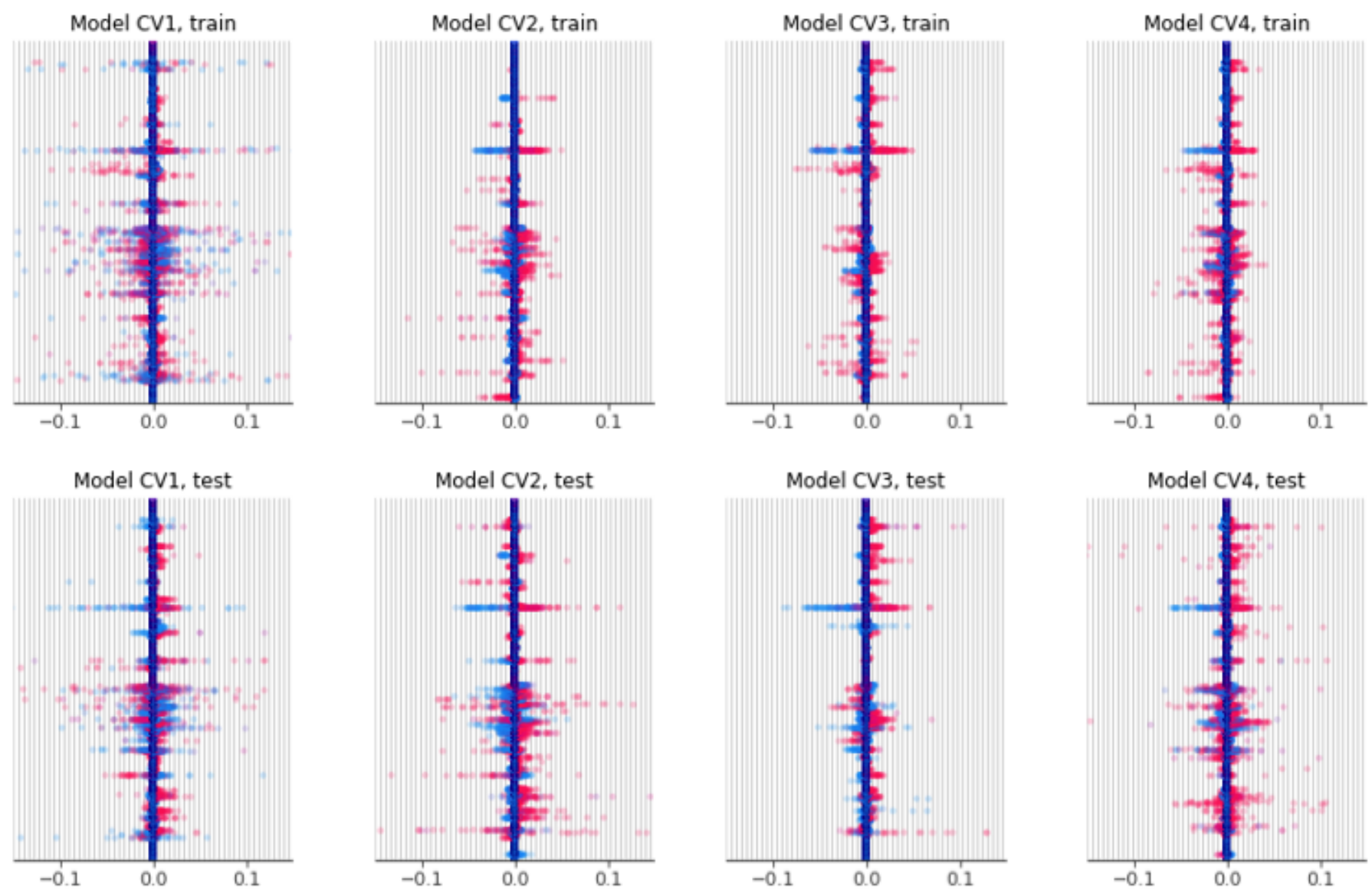

\section{Figure 3}

Beeswarm summary plots on train and test data. This plot combines feature importance and feature effects. Every feature (VOC) is represented as a row on the y-axis (3400 total) and SHAP values are on the $x$-axis (multiple VOC may overlap at a single index). Each dot represents a Shapley value for a given sample prediction. The color intensity shows the magnitude of importance of each feature. 

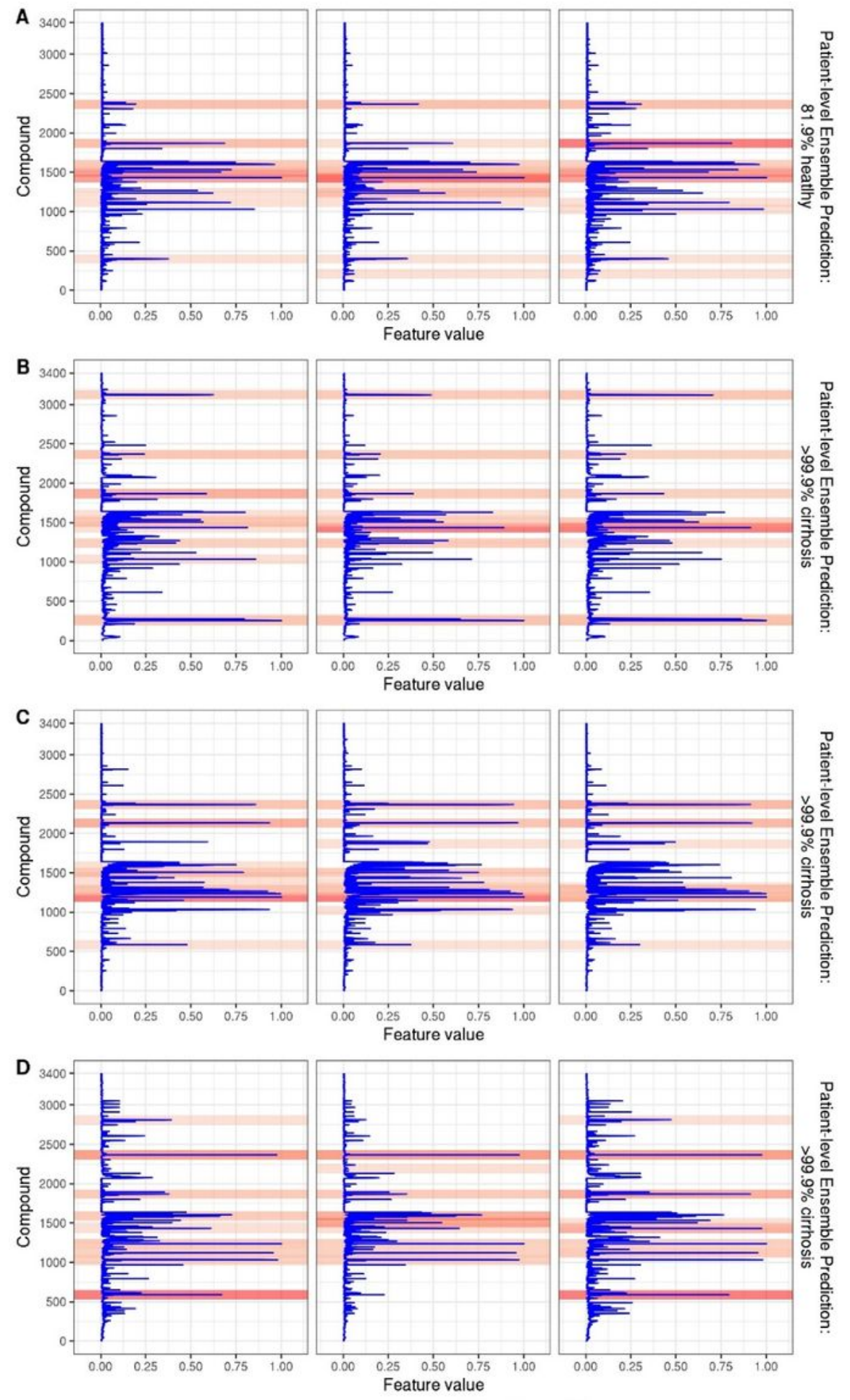

Number of CV models

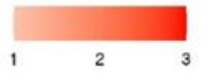

\section{Figure 4}

Patient breath samples with overlayed heatmaps which identify the 5 most important peaks from each CV model (up to 20 peaks total) in the classification of liver cirrhosis for a healthy control (A), and 3 individuals with stage I (B), stage II (C), and stage III (D) cirrhosis, respectively. Compounds are represented by indices on the $y$-axis and VOC signal value is on the $\mathrm{x}$-axis; darker shading indicates the feature was selected by multiple CV models 


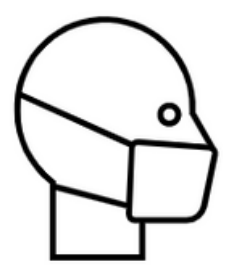

Step 1. 1L sample of patient breath is collected in a mask

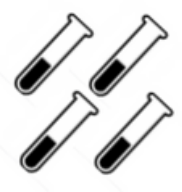

Step 2. Four technical replicates are derived from the breath sample

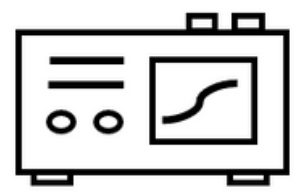

Step 3. Volatolomic profile analyzed from

each sample

independently using TD-GC-FAIMS

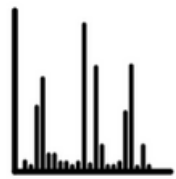

Step 4. Volatolomic profile normalized to room-air measurement and preprocessed

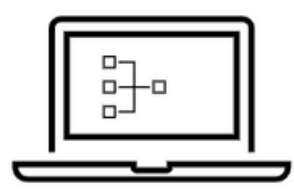

Step 5. Deep learning algorithm trained to predict cirrhosis on postprocessed signal

\section{Figure 5}

Flow-chart of sample collection and analysis. 


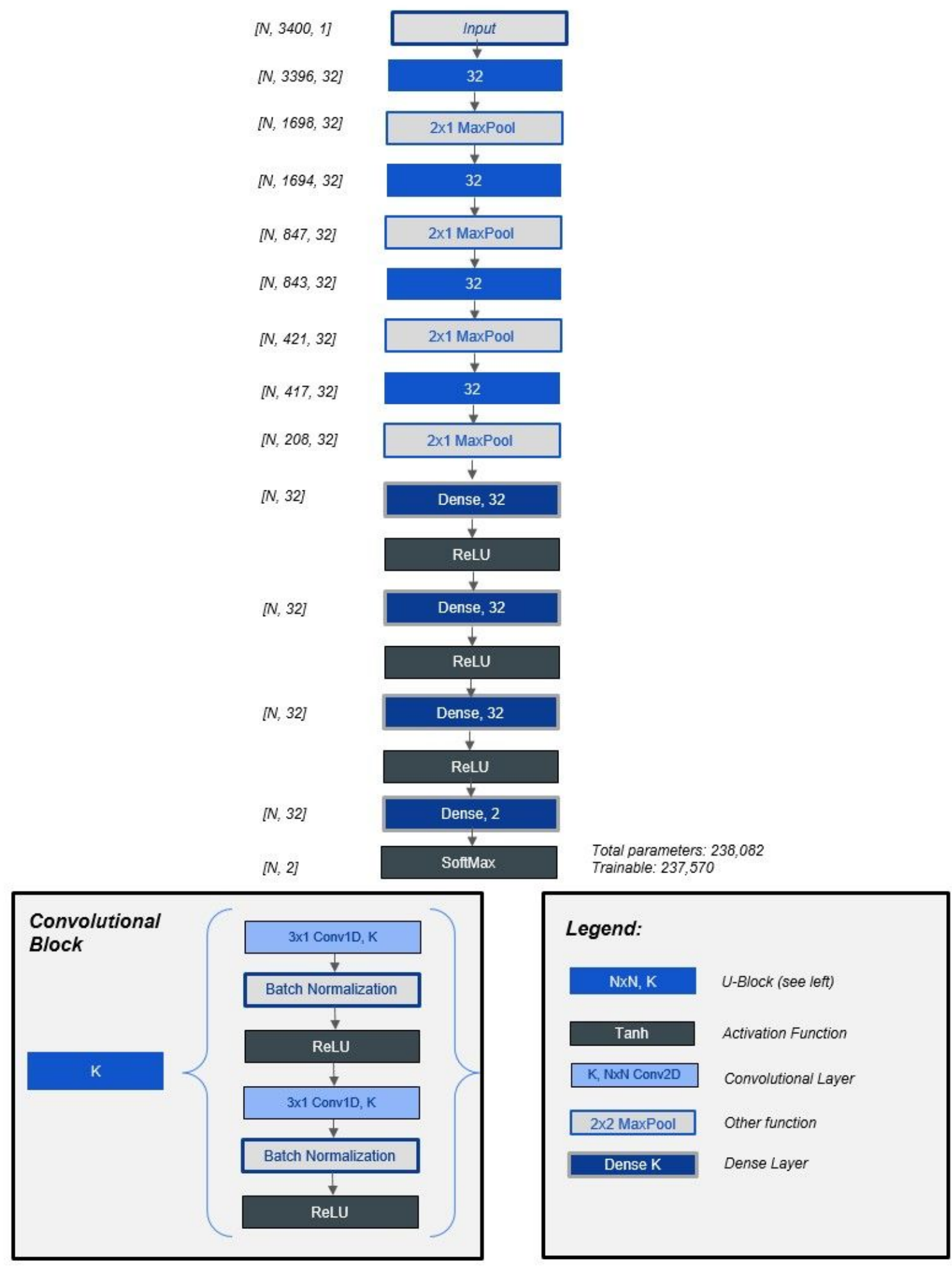

Figure 6

Diagram of custom CNN model architecture.

\section{Supplementary Files}

This is a list of supplementary files associated with this preprint. Click to download. 
- Supplementaryinformation.docx

Page 22/22 